International Journal of Pure and Applied Mathematics

Volume $91 \quad$ No. $3 \quad 2014,313-319$

ISSN: 1311-8080 (printed version); ISSN: 1314-3395 (on-line version)

url: http://www.ijpam.eu

doi: http://dx.doi.org/10.12732/ijpam.v91i3.4

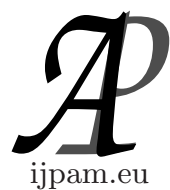

\title{
POISSON APPROXIMATION FOR THE NUMBER OF OPIES OF A FIXED GRAPH IN A RANDOM d-REGULAR GRAPH
}

\author{
Mana Donganont \\ Department of Mathematics \\ School of Science University of Phayao \\ Phayao, THAILAND
}

\begin{abstract}
Let $W$ be the number of copies of a fixed graph $H$ in a random dregular graph $\mathbb{G}_{n, d}$. In this paper, we give the bound on Poisson approximation of $W$ by using the Stein-Chen method.
\end{abstract}

Key Words: random d-regular graph, Stein's method, coupling approach

\section{Introduction}

Random graphs, introduced by P. Erdös and Rényi[5], still continue to attract a huge amount of research and interest in the communities of Graph Theory, Theoretical Computer Science and Discrete Mathematics.

There exist several models of random graphs. The most famous is the $\mathbb{G}(n, p)$ random graph, defined on $n$ labeled vertices $1,2, . ., n$ and the edges added randomly such that each of the $\left(\begin{array}{l}n \\ 2\end{array}\right)$ possible edges exists with probability $p, 0<p<1$. Other models have also been quite a lot investigated ; $\mathbb{G}(n, M)$ produced by randomly and equiprobably selecting an element of the class of graphs on $n$ vertices having $M$ edges.

This work is a survey of that part of random graph theory in which the degrees of vertices are restricted. Such work concentrates on regular graphs as the most interesting examples, and the results on regular graphs often extend easily to more general degree sequences. Let $1 \leq d \leq n-1$ be two positive

Received: October 23, 2013

(c) 2014 Academic Publications, Ltd. url: www.acadpubl.eu 
integer, a random graph $\mathbb{G}_{n, d}$ is obtained by sampling uniformly at random over the set of all simple $d$-regular graph is on a fixed set of $n$ vertices. We refer the readers to Wormald's survey [3] for more information (both historical and technical) about this model. As usual, $\mathbb{G}_{n, d}$ denotes the random $d$-regular graph with $n$ labeled vertices and for each vertex has the same degree $d$. For each $H=(V(H), E(H))$ be a graph, we use the notation $v_{H}=|V(H)|$ and $e_{H}=|E(H)|$ for the number of vertices and edges, respectively. If a subgraph $H^{\prime}$ of a graph $F$ is isomorphic to graph $H$, then $H^{\prime}$ is called a copy of $H$ in $F$.

Let

$$
\Gamma=\left\{i=:\left\{i_{1}, i_{2}, \ldots, i_{v_{H}}\right\} \mid 1 \leq i_{1}<\ldots<i_{v_{H}} \leq n\right\}
$$

be the set of all possible combinations of $v_{H}$ vertices. For each $i \in \Gamma$, we define the indicator random variable

$$
X_{i}=\left\{\begin{aligned}
1 & \text { if there is a copy of } H \text { in } \mathbb{G}_{n, d} \text { that spans the vertices } \\
& i=\left(i_{1}, \ldots, i_{v_{H}}\right), \\
0 & \text { otherwise, }
\end{aligned}\right.
$$

and

$$
W=\sum_{i \in \Gamma} X_{i} .
$$

Then $W$ is the number of copies of $H$ in $\mathbb{G}_{n, d}$.

In 2007, Jeong Han Kim, Benny Sodakov and Van Vu [2] proved that the distribution function of $W$ can be approximate by Poisson distribution as the following result.

Theorem 1.1. Let $H$ be a strictly balanced graph with $v$ vertices and $e \geq$ $v$ edges. Let $A u t(H)$ be the number of automorphisms of $H$ and let $W$ be the number of copies of $H$ in a random d-regular graph $\mathbb{G}_{n, d}$. If $(d-1) n^{-1+\frac{v}{e}} \rightarrow c$ for some positive constant $c$, then $W$ converges to Poi $i_{\lambda}$, the Poisson distribution with mean $\lambda=\frac{c^{e}}{\operatorname{Aut}(H)}$.

Next, in 2010, Mana Donganont and Angkana Boonyued [9] showed that the distribution function of $W$ can be approximate by Poisson distribution and the error term of this approximation by using Stein-Chen method and Local approach the result as following.

Theorem 1.2. [9] Let $H$ be a fixed graph with $v_{H}$ vertices and $e_{H} \geq v_{H}$ edges and let $W$ be the number of copies of $H$ in $\mathbb{G}_{n, d}$. If $d=n^{\delta}$ where $\delta<1$ such that $(1-\delta) e_{H} \geq 2 v_{H}$ then there exists a constant $C_{H}>0$ such that

$$
\sup _{A \subseteq \mathbb{R}}\left|P(W \in A)-\operatorname{Poi}_{\lambda}(A)\right| \leq \frac{C_{H}}{n^{(1-\delta) e_{H}-2 v_{H}}} .
$$


In this work, we use the Stein-Chen and Coupling method to approximate $W$, the number of copies of $H$ in $\mathbb{G}_{n, d}$, and show that the error term of this approximation is better than that of Mana Donganont and Angkana Boonyued [9]. The result as following.

This paper is organized as follows. In Section 2, we introduce the SteinChen method for Poisson approximation and local approach which use in the proof of main result in Section 3.

Throughout this paper, $C_{H}$ stands for an absolute constant depend on $H$ and which possible different values in different places.

\section{Stein-Chen and Coupling Method}

In 1972, Stein [1] gave a new technique to find a bound in the normal approximation to a distribution of a sum of dependent random variables. His technique was relied instead on the elementary differential equation. In 1975, Chen $[4,5]$ applied Stein's idea to the Poisson case. The central idea of the Stein-Chen method is the difference equation

$$
I_{A}(j)-\mathcal{P}_{\lambda}(A)=\lambda g_{\lambda, A}(j+1)-j g_{\lambda, A}(j), j \in \mathbb{N} \cup\{0\}
$$

where $\lambda>0$ and $A \subseteq \mathbb{N} \cup\{0\}$ and $I_{A}: \mathbb{N} \cup\{0\} \rightarrow \mathbb{R}$ is defined by

$$
I_{A}(w)= \begin{cases}1 & ; w \in A \\ 0 & ; w \notin A .\end{cases}
$$

The equation (1) is called Stein's equation for Poisson distribution function and its solution is

$$
g_{\lambda, A}(w)= \begin{cases}(w-1) ! \lambda^{-w} e^{\lambda}\left[\mathcal{P}_{\lambda}\left(I_{A \cap C_{w-1}}\right)-\mathcal{P}_{\lambda}\left(I_{A}\right) \mathcal{P}_{\lambda}\left(I_{C_{w-1}}\right)\right] & ; w \geq 1, \\ 0 & ; w=0\end{cases}
$$

where

$$
C_{w-1}=\{0,1, \ldots, w-1\} \text { and } \mathcal{P}_{\lambda}\left(I_{A}\right)=e^{-\lambda} \sum_{l=0}^{\infty} I_{A}(l) \frac{\lambda^{l}}{l !} \cdot[7]
$$

By substituting $j$ and $\lambda$ in (1) by any integer-valued random variable $W$ and $\lambda=\mathrm{E}(W)$, we have

$$
P(W \in A)-P o i_{\lambda}(A)=\mathrm{E}\left(\lambda g_{\lambda, A}(W+1)\right)-\mathrm{E}\left(W g_{\lambda, A}(W)\right) .
$$

So far $W$ could be $\sum_{i \in \Gamma} X_{i}$ and $\lambda=\mathbb{E}(W)=\sum_{i \in \Gamma} p_{i}$ where $p_{i}=\mathbb{E}\left(X_{i}\right)=$ $P\left(X_{i}=1\right)$. 
In 1992, Barbour, Holst and Janson[7] constructed coupling random variable $W_{i}$ and used Stein-Chen method to find the bound in Poisson approximation of $W$. They assumed that for each $i$ the distribution $L\left(W_{i}\right)$ of $W_{i}$ equals to the conditional distribution $L\left(W-X_{i} \mid X_{i}=1\right)$ and gave the fundamental theorem as follows:

Theorem 2.1. If $W$ and $W_{i}$ are defined as above, then

$$
\left|P(W \in A)-P \operatorname{Poi}_{\lambda}(A)\right| \leq\left\|g_{\lambda, A}\right\| \sum_{i \in \Gamma} p_{i} \mathbb{E}\left(\left|W-W_{i}\right|\right)
$$

where $\left\|g_{\lambda, A}\right\|:=\sup _{w}\left[g_{\lambda, A}(w+1)-g_{\lambda, A}(w)\right]$.

In 2006, Santiwipanont and Teerapabolarn [8] proved that for any subset $A$ of $\{0,1, \ldots, n\}$,

$$
\left\|g_{\lambda, A}\right\| \leq \lambda^{-1} \min \left\{1, \lambda, \frac{\triangle(\lambda)}{M_{A}+1}\right\}
$$

where

$$
\triangle(\lambda)= \begin{cases}e^{\lambda}+\lambda-1 & \text { if } \lambda^{-1}\left(e^{\lambda}-1\right) \leq M_{A}, \\ 2\left(e^{\lambda}-1\right) & \text { if } \lambda^{-1}\left(e^{\lambda}-1\right)>M_{A},\end{cases}
$$

and

$$
M_{A}= \begin{cases}\max \left\{w \mid C_{w} \subseteq A\right\} & \text { if } 0 \in A, \\ \min \{w \mid w \in A\} & \text { if } 0 \notin A .\end{cases}
$$

In next section, we will use Theorem 2.1 to prove our main result.

\section{Proof of Main Result}

In this section we prove the our main theorem. Let $H$ be a fixed graph with $v_{H}$ vertices and $e_{H}$ edges such that $e_{H} \geq v_{H}$ and $A \subseteq \mathbb{N}$. By (3), it suffices to bound $\mathbb{E}\left|W-W_{i}\right|$ for any $i \in\{1,2, \ldots, n\}$ where the distribution of $W_{i}$ equals to the conditional distribution of $W-X_{i}$ given $X_{i}=1$.

Let

$$
\Gamma=\left\{i=:\left\{i_{1}, i_{2}, \ldots, i_{v_{H}}\right\} \mid 1 \leq i_{1}<\ldots<i_{v_{H}} \leq n\right\},
$$

and

$$
X_{i}=\left\{\begin{aligned}
1 & \text { if there is a copy of } H \text { in } \mathbb{G}_{n, d} \text { that spans the vertices } \\
& i=\left(i_{1}, \ldots, i_{v_{H}}\right), \\
0 & \text { otherwise }
\end{aligned}\right.
$$


We divide $\Gamma \backslash\{i\}$ into two subsets as follow

$$
\Gamma_{i}^{w}=\{j \in \Gamma \backslash\{i\} \mid i \cap j=\phi\}, \Gamma_{i}^{s}=\{j \in \Gamma \backslash\{i\} \mid i \cap j \neq \phi\}
$$

and define $Z_{i}=\sum_{j \in \Gamma_{i}^{s}} X_{j}$ and $W_{i}=\sum_{j \in \Gamma_{i}^{w}} X_{j}$.

J.H. Kim, B. Sodakov and V. Vu [2] show that the expectation of the number of copies of a graph $H$ in $\mathbb{G}_{n, d}$ is asymptotically the same as the expectation of the number of copies of $H$ in $\mathbb{G}(n, p)$, where $p=\frac{d}{n}$.

Then

$$
\mathbb{E}\left(X_{i}\right)=p_{i}=P\left(X_{i}=1\right)=\frac{v_{H} !}{A u t(H)}\left(\frac{d}{n}\right)^{e_{H}}\left(1-\frac{d}{n}\right)^{\frac{n d}{2}-e_{H}}
$$

for all $i \in \Gamma$ and

$$
\lambda=\mathbb{E}(W)=\left(\begin{array}{c}
n \\
v_{H}
\end{array}\right) P\left(X_{i}=1\right)=\left(\begin{array}{c}
n \\
v_{H}
\end{array}\right) \frac{v_{H} !}{A u t(H)}\left(\frac{d}{n}\right)^{e_{H}}\left(1-\frac{d}{n}\right)^{\frac{n d}{2}-e_{H}},
$$

where $\frac{v_{H} !}{A u t(H)}$ is a number copies of $H$ which spans the vertex $i$.

By definition of $Z_{i}$, we have

$$
\mathbb{E}\left(Z_{i}\right)=\mathbb{E}\left(\sum_{j \in \Gamma_{i}^{s}} X_{j}\right)=\sum_{j \in \Gamma_{i}^{s}} \mathbb{E}\left(X_{j}\right)=p_{i}\left[\left(\begin{array}{c}
n \\
v_{H}
\end{array}\right)-\left(\begin{array}{c}
n-v_{H} \\
v_{H}
\end{array}\right)-1\right] .
$$

Now, we consider $\mathbb{E}\left(X_{i} X_{j}\right)$ for $j \in \Gamma_{i}^{s}$.

Let $H^{\prime}$ and $H^{\prime \prime}$ be copies of $H$ in $\mathbb{G}_{n, d}$ such that spans by the vertices $i$ and $j$ respectively.

Case 1. $E\left(H^{\prime}\right) \cap E\left(H^{\prime \prime}\right)=\phi$.

$$
\mathbb{E}\left(X_{i} X_{j}\right)=P\left(X_{i}=1, X_{j}=1\right)=\left[\frac{v_{H} !}{A u t(H)}\right]^{2}\left[\left(\frac{d}{n}\right)^{e_{H}}\left(1-\frac{d}{n}\right)^{\frac{n d}{2}-e_{H}}\right]^{2} .
$$

Case 2. $E\left(H^{\prime}\right) \cap E\left(H^{\prime \prime}\right) \neq \phi$.

Let $F$ be a subgraph of $H$ such that $F$ isomorphic to $H^{\prime} \cap H^{\prime \prime}$. Then we have

$$
\begin{aligned}
\mathbb{E}\left(X_{i} X_{j}\right) & =P\left(X_{i}=1, X_{j}=1\right) \\
& =\left[\frac{v_{H} !}{A u t(H)}\right]^{2}\left(\frac{d}{n}\right)^{2 e_{H}-e_{F}}\left(1-\frac{d}{n}\right)^{\frac{n d}{2}-\left(2 e_{H}-e_{F}\right)} .
\end{aligned}
$$


From (7) and (8), we have

$$
\begin{aligned}
& \mathbb{E}\left(X_{i} X_{j}\right)= \\
& \left(\frac{v_{H} !}{A u t(H)}\right)^{2}\left[\left(\left(\frac{d}{n}\right)^{e_{H}}\left(1-\frac{d}{n}\right)^{\frac{n d}{2}-e_{H}}\right)^{2}+\left(\frac{d}{n}\right)^{2 e_{H}-e_{F}}\left(1-\frac{d}{n}\right)^{\frac{n d}{2}-\left(2 e_{H}-e_{F}\right)}\right] .
\end{aligned}
$$

Thus,

$$
\begin{aligned}
\mathbb{E}\left(X_{i} Z_{i}\right) & =\mathbb{E}\left(X_{i} \sum_{i \in \Gamma_{i}^{s}} X_{j}\right) \\
& =\sum_{i \in \Gamma_{i}^{s}} \mathbb{E}\left(X_{i} X_{j}\right) \\
& \leq C_{H}\left(\begin{array}{c}
n \\
v_{H}
\end{array}\right)\left[\left(\left(\frac{d}{n}\right)^{e_{H}}\left(1-\frac{d}{n}\right)^{\frac{n d}{2}}-e_{H}\right)^{2}+\left(\frac{d}{n}\right)^{2 e_{H}-e_{F}}\left(1-\frac{d}{n}\right)^{\frac{n d}{2}}-\left(2 e_{H}-e_{F}\right)\right],
\end{aligned}
$$

where $C_{H}$ stands for an absolute constant depend on $H$.

By Theorem 2.1, (5), (6) and (9), we get $\sup _{A \subset \mathbb{Z}_{+}}\left|P(W \in A)-P o i_{\lambda}(A)\right|$

$$
\begin{aligned}
& \leq k_{2}(\lambda) \sum_{i \in \Gamma}\left(p_{i} \mathbb{E}\left(X_{i}+Z_{i}\right)+\mathbb{E}\left(X_{i} Z_{i}\right)\right) \\
& =k_{2}(\lambda) \sum_{i \in \Gamma}\left[p_{i} \mathbb{E}\left(X_{i}\right)+p_{i} \mathbb{E}\left(Z_{i}\right)+\mathbb{E}\left(X_{i} Z_{i}\right)\right] \\
\leq & k_{2}(\lambda) C_{H}\left(\begin{array}{c}
n \\
v_{H}
\end{array}\right)\left\{\left[\left(\left(\frac{d}{n}\right)^{e_{H}}\left(1-\frac{d}{n}\right)^{\frac{n d}{2}}-e_{H}\right)^{2}+\left(\begin{array}{c}
n \\
v_{H}
\end{array}\right)\left(\left(\frac{d}{n}\right)^{e_{H}}\left(1-\frac{d}{n}\right)^{\frac{n d}{2}-e_{H}}\right)^{2}\right]\right. \\
+ & \left.\left(\begin{array}{c}
n \\
v_{H}
\end{array}\right)\left[\left(\left(\frac{d}{n}\right)^{e_{H}}\left(1-\frac{d}{n}\right)^{\frac{n d}{2}-e_{H}}\right)^{2}+\left(\frac{d}{n}\right)^{2 e_{H}-e_{F}}\left(1-\frac{d}{n}\right)^{\frac{n d}{2}}-\left(2 e_{H}-e_{F}\right)\right]\right\} .
\end{aligned}
$$

By the fact that $e_{F} \leq e_{H}$ and $\left(1-\frac{d}{n}\right)^{\frac{n d}{2}}-e_{H}$ converges to some positive constant when $d=n^{\delta}$ for $\delta<1$ such that $(1-\delta) e_{H} \geq 2 v_{H}$, we have for $d=n^{\delta}$

$$
\begin{aligned}
& \sup _{A \subset \mathbb{Z}_{+}}\left|P(W \in A)-\operatorname{Poi}_{\lambda}(A)\right| \\
\leq & C_{H}\left(n^{v_{H}}\right)\left[\frac{1}{n^{2(1-\delta) e_{H}}}+n^{v} \frac{1}{n^{2(1-\delta) e_{H}}}+n^{v_{H}} \frac{1}{n^{2(1-\delta) e_{H}}}+n^{v_{H}} \frac{1}{n^{(1-\delta)\left(2 e_{H}-e_{F}\right)}}\right] \\
= & C_{H}\left[\left(\frac{1}{n^{2(1-\delta) e_{H}-v_{H}}}+\frac{1}{n^{2(1-\delta) e_{H}-2 v_{H}}}+\frac{1}{n^{2(1-\delta) e_{H}-2 v_{H}}}+\frac{1}{n^{(1-\delta)\left(2 e_{H}-e_{F}\right)-2 v_{H}}}\right]\right. \\
\leq & \frac{C_{H}}{n^{(1-\delta) e_{H}-2 v_{H}}} .
\end{aligned}
$$

This complete the proof. 


\section{References}

[1] A.D. Barbour, Louis H.Y. Chen, An Introduction to Stein's Method, Singapore university Press and world scientific Publishing Co. Pte. Ltd, 2005.

[2] J.H. Kim, B. Sodakov, V. Vu, Small subgraphs of random regular graphs, Math. Debrecen, 6 (1959), 290-297.

[3] N. Wormald, Models of random regular graphs, In: Surveys in Combinatorics, Proceeding Cambridge 1999, London Mathematical Society, Lecture Note Ser., Vol. 276, Cambridge University Press, Cambridge, 1999, pp. 239-298.

[4] L.H.Y. Chen (1975), Poisson approximation for dependent trials, Annals of Probability, 3, 534-545.

[5] P. Erdős, A. Rényi, On the evolution of random graphs, Publ. Math. Inst. Hungar. Acad. Sci., 5 (1960) 17-61.

[6] C.M. Stein, A bound for the error in normal approximation to the distribution of a sum of dependent random variables, In: Proc. Sixth Berkeley Sympos., Math. Statist. Probab., 3 (1972), 583-602.

[7] A.D. Barbour, L. Holst, S. Janson, Poisson Approximation, Oxford Studies in probability 2, Clarendon Press, Oxford, 1992.

[8] T. Santiwipanont, K. Teerapabolarn, Two formulas of non-uniform bounds on Poisson approximation for dependent indicators, Thai Journal of Mathematics (2006).

[9] M. Donganont, A. Boonyued, The bound on Poisson approximation of the number of copies of a fixed graph in a random $d$-regular graph, In: Proc. Sixth Berkeley Sympos, Math. Statist. Probab., 3 (2012), 583-602. 
